\title{
Research Article \\ On the Convergence of Solutions of Certain Third-Order Differential Equations
}

\author{
Ercan Tunç \\ Department of Mathematics, Faculty of Arts and Sciences, Gaziosmanpaşa University, 60240-Tokat, Turkey \\ Correspondence should be addressed to Ercan Tunç, ercantunc72@yahoo.com \\ Received 29 December 2008; Revised 24 March 2009; Accepted 5 April 2009 \\ Recommended by Leonid Shaikhet \\ We establish sufficient conditions for the convergence of solutions of a certain third-order nonlinear \\ differential equations. By constructing a Lyapunov function as the basic tool, some results which \\ exist in the relevant literature are generalized. \\ Copyright (C) 2009 Ercan Tunç. This is an open access article distributed under the Creative \\ Commons Attribution License, which permits unrestricted use, distribution, and reproduction in \\ any medium, provided the original work is properly cited.
}

\section{Introduction}

As well known, in the investigation of qualitative behaviors of solutions, stability, convergence, boundedness, oscillation, and so forth of solutions are very important problems in theory and applications of differential equations. For example, in applied sciences, some practical problems concerning mechanics, the engineering technique fields, economy, control theory, physics, chemistry, biology, medicine, atomic energy, information theory, and so forth are associated with certain higher-order linear or nonlinear differential equations. Ever since Lyapunov [1] proposed his famous theory on the stability of motion, For some papers published on the qualitative behaviors of solutions of nonlinear second-and third-order differential equations, the readers can referee to the papers of Afuwape and Omeike [2,3], Ezeilo [4, 5], Meng [6], Tejumola [7, 8], Tunç [9-11], Omeike [12], and the references listed in these papers as well as one can refer to the books of Reissig et al. [13, 14]. The motivation for the present work has been inspired basically by the paper of Afuwape and Omeike [2] and the papers listed above. Our aim here is to extend the results established by Afuwape and Omeike [2] to nonlinear differential equation (1.4) for the convergence of all solutions of this equation. In 2008, Afuwape and Omeike [2] considered third-order nonlinear differential equations of the form

$$
\dddot{x}+a \ddot{x}+g(\dot{x})+h(x)=p(t, x, \dot{x}, \ddot{x}),
$$


and by introducing a Lyapunov function they discussed the convergence of solutions for this equation. During establishment of the results, Afuwape and Omeike [2] defined the following relations with respect to the functions $g$ and $h$ :

$$
0<b \leq \frac{g\left(y_{2}\right)-g\left(y_{1}\right)}{y_{2}-y_{1}} \leq b_{0}<\infty,
$$

for any pair of constants $y_{2}, y_{1}\left(y_{2} \neq y_{1}\right)$ and

$$
0<\delta \leq \frac{h\left(x_{2}\right)-h\left(x_{1}\right)}{x_{2}-x_{1}} \leq k a b
$$

for any pair of constants $x_{2}, x_{1}\left(x_{2} \neq x_{1}\right)$, where $k<1$ is a positive constant.

In this paper, we consider nonlinear differential equation of the form

$$
\dddot{x}+f(\ddot{x})+g(\dot{x})+h(x)=p(t, x, \dot{x}, \ddot{x}),
$$

where the functions $f, g, h$, and $p$ are continuous in their respective arguments, with the functions $f, g$, and $h$ are not necessarily differentiable. In addition to (1.2) and (1.3) we assume that

$$
0<a \leq \frac{f\left(z_{2}\right)-f\left(z_{1}\right)}{z_{2}-z_{1}} \leq a_{0}<\infty,
$$

for any pair of constants $z_{2}, z_{1}\left(z_{2} \neq z_{1}\right)$.

By convergence of solutions we mean, any two solutions $x_{1}(t), x_{2}(t)$ of (1.4) are said to converge to each other if

$$
x_{2}(t)-x_{1}(t) \longrightarrow 0, \quad \dot{x}_{2}(t)-\dot{x}_{1}(t) \longrightarrow 0, \quad \ddot{x}_{2}(t)-\ddot{x}_{1}(t) \longrightarrow 0
$$

as $t \rightarrow \infty$.

\section{Main Results}

The following results are established.

Theorem 2.1. Suppose that $f(0)=g(0)=h(0)$, and that

(i) there are constants $a>0, a_{0}>0$ such that $f(z)$ satisfies inequalities (1.5),

(ii) there are constants $b>0, \quad b_{0}>0$ such that $g(y)$ satisfies inequalities (1.2),

(iii) there are constants $\delta>0, k<1$ such that for any $\xi, \eta(\eta \neq 0)$, the incrementary ratio for $h$ satisfies

$$
\frac{(h(\xi+\eta)-h(\xi))}{\eta} \text { lies in } I_{0}
$$

with $I_{0}=[\delta, k a b]$, 
Discrete Dynamics in Nature and Society

(iv) there is a continuous function $\phi(t)$ such that

$$
\left|p\left(t, x_{2}, y_{2}, z_{2}\right)-p\left(t, x_{1}, y_{1}, z_{1}\right)\right| \leq \phi(t)\left\{\left|x_{2}-x_{1}\right|+\left|y_{2}-y_{1}\right|+\left|z_{2}-z_{1}\right|\right\}
$$

holds for arbitrary $t, x_{1}, y_{1}, z_{1}, x_{2}, y_{2}, z_{2}$, and satisfies

$$
\int_{0}^{t} \phi^{v}(\tau) d \tau \leq D_{1} t
$$

for some constant $D_{1}>0$, where $v$ is a constant in the range $1 \leq v \leq 2$.

Then all solutions of (1.4) converge.

A very important step in the proof of Theorem 2.1 will be to give estimate for any two solutions of (1.4). This in itself, being of independent interest, is giving as follows.

Theorem 2.2. Let $x_{1}(t), x_{2}(t)$ be any two solutions of (1.4). Suppose that all the conditions of Theorem 2.1 are satisfied, then for each fixed $v$, in the range $1 \leq v \leq 2$, there exist constants $D_{2}, D_{3}$, and $D_{4}$ such that for $t_{2} \geq t_{1}$,

$$
S\left(t_{2}\right) \leq D_{2} S\left(t_{1}\right) \exp \left\{-D_{3}\left(t_{2}-t_{1}\right)+D_{4} \int_{t_{1}}^{t_{2}} \phi^{v}(\tau) d \tau\right\},
$$

where

$$
S(t)=\left\{\left[x_{2}(t)-x_{1}(t)\right]^{2}+\left[\dot{x}_{2}(t)-\dot{x}_{1}(t)\right]^{2}+\left[\ddot{x}_{2}(t)-\ddot{x}_{1}(t)\right]^{2}\right\}
$$

We have the following corollaries when $x_{1}(t)=0$ and $t_{1}=0$.

Corollary 2.3. Suppose that $p=0$ in (1.4) and suppose further that conditions (i), (ii), and (iii) of Theorem 2.1 hold, then the trivial solution of (1.4) is exponentially stable in the large.

Also, if we put $\xi=0$ in (2.1) with $\eta(\eta \neq 0)$ arbitrary, we get the following.

Corollary 2.4. If $p \neq 0$ and hypotheses (i), (ii), and (iii) of Theorem 2.1 hold for arbitrary $\eta(\eta \neq 0)$, and $\xi=0$, then there exists a constant $D_{5}>0$ such that every solution $x(t)$ of (1.4) satisfies

$$
|x(t)| \leq D_{5}, \quad|\dot{x}(t)| \leq D_{5}, \quad|\ddot{x}(t)| \leq D_{5} .
$$

\section{Preliminary Results}

On setting $\dot{x}=y, \dot{y}=z$, (1.4) can be replaced by an equivalent system

$$
\dot{x}=y, \quad \dot{y}=z, \quad \dot{z}=-f(z)-g(y)-h(x)+p(t, x, y, z) .
$$


Let $\left(x_{i}(t), y_{i}(t), z_{i}(t)\right), i=1,2$, be any two solutions of (3.1) such that

$$
\begin{gathered}
a \leq \frac{f\left(z_{2}\right)-f\left(z_{1}\right)}{z_{2}-z_{1}} \leq a_{0} \quad\left(z_{2} \neq z_{1}\right), \\
b \leq \frac{g\left(y_{2}\right)-g\left(y_{1}\right)}{y_{2}-y_{1}} \leq b_{0} \quad\left(y_{2} \neq y_{1}\right), \\
\delta \leq \frac{h\left(x_{2}\right)-h\left(x_{1}\right)}{x_{2}-x_{1}} \leq k a b \quad\left(x_{2} \neq x_{1}\right),
\end{gathered}
$$

where $a_{0}, a, b_{0}, b, \delta$, and $k$ are finite constants, and $k$ will be determined later.

Our investigation rests mainly on the properties of the function, $W=W\left(x_{2}-x_{1}, y_{2}-\right.$ $y_{1}, z_{2}-z_{1}$ ) defined by

$$
\begin{aligned}
& 2 W=\beta(1-\beta) b^{2}\left(x_{2}-x_{1}\right)^{2}+\beta b\left(y_{2}-y_{1}\right)^{2}+\alpha b a^{-1}\left(y_{2}-y_{1}\right)^{2} \\
&+\alpha a^{-1}\left(z_{2}-z_{1}\right)^{2}+\left\{\left(z_{2}-z_{1}\right)+a\left(y_{2}-y_{1}\right)+(1-\beta) b\left(x_{2}-x_{1}\right)\right\}^{2},
\end{aligned}
$$

where $0<\beta<1$ and $\alpha>0$ are constants.

Following the argument used in [5], we can easily verify the following for $W$.

Lemma 3.1. (i) $W(0,0,0)=0$.

(ii) There exist finite positive constants $D_{6}, D_{7}$ such that

$$
D_{6}\left\{\left(x_{2}-x_{1}\right)^{2}+\left(y_{2}-y_{1}\right)^{2}+\left(z_{2}-z_{1}\right)^{2}\right\} \leq W \leq D_{7}\left\{\left(x_{2}-x_{1}\right)^{2}+\left(y_{2}-y_{1}\right)^{2}+\left(z_{2}-z_{1}\right)^{2}\right\}
$$

where

$$
D_{6}=\frac{1}{2} \min \left\{\beta(1-\beta) b^{2}, b\left(\beta+\alpha a^{-1}\right), \alpha a^{-1}\right\}
$$

and using the inequality $|x||y| \leq(1 / 2)\left(x^{2}+y^{2}\right)$,

$$
D_{7}=\frac{1}{2} \max \left\{b(1-\beta)(1+b+a), b\left(\beta+\alpha a^{-1}\right)+a(1+a+b(1-\beta)), 1+\alpha a^{-1}+a+b(1-\beta)\right\} .
$$

If we define the function $W(t)$ by

$$
W\left(x_{2}(t)-x_{1}(t), y_{2}(t)-y_{1}(t), z_{2}(t)-z_{1}(t)\right)
$$

and using the fact that the solutions $\left(x_{i}, y_{i}, z_{i}\right), i=1,2$, satisfy (3.1), then $S(t)$ as defined in (2.5) becomes

$$
S(t)=\left\{\left[x_{2}(t)-x_{1}(t)\right]^{2}+\left[y_{2}(t)-y_{1}(t)\right]^{2}+\left[z_{2}(t)-z_{1}(t)\right]^{2}\right\} .
$$


Lemma 3.2. Assume that the conditions (i), (ii), and (iii) of Theorem 2.1 are satisfied. Then, there exist positive finite constants $D_{8}$ and $D_{9}$ such that

$$
\frac{d W}{d t} \leq-2 D_{8} S+D_{9} S^{1 / 2}|\theta|
$$

where $\theta=p\left(t, x_{2}, y_{2}, z_{2}\right)-p\left(t, x_{1}, y_{1}, z_{1}\right)$.

\section{Proof of Lemma 3.2}

Differentiating the function $W$ in (3.3) along the system (3.1) we obtain

$$
\dot{W}=\frac{d W}{d t}=-W_{1}-W_{2}-W_{3}-W_{4}-W_{5}-W_{6}-W_{7}+W_{8}
$$

in which

$$
\begin{aligned}
& W_{1}=\left\{r_{1} b(1-\beta) H\left(x_{2}, x_{1}\right)\left(x_{2}-x_{1}\right)^{2}+\eta_{1} a\left[G\left(y_{2}, y_{1}\right)-b(1-\beta)\right]\left(y_{2}-y_{1}\right)^{2}\right. \\
& \left.+\xi_{1} \alpha a^{-1} F\left(z_{2}, z_{1}\right)\left(z_{2}-z_{1}\right)^{2}+\left(F\left(z_{2}, z_{1}\right)-a\right)\left(z_{2}-z_{1}\right)^{2}\right\}, \\
& W_{2}=\left\{r_{2} b(1-\beta) H\left(x_{2}, x_{1}\right)\left(x_{2}-x_{1}\right)^{2}+\xi_{2} \alpha a^{-1} F\left(z_{2}, z_{1}\right)\left(z_{2}-z_{1}\right)^{2}\right. \\
& \left.+\left(1+\alpha a^{-1}\right)\left(x_{2}-x_{1}\right)\left(z_{2}-z_{1}\right) H\left(x_{2}, x_{1}\right)\right\}, \\
& W_{3}=\left\{r_{3} b(1-\beta) H\left(x_{2}, x_{1}\right)\left(x_{2}-x_{1}\right)^{2}+\eta_{2} a\left[G\left(y_{2}, y_{1}\right)-b(1-\beta)\right]\left(y_{2}-y_{1}\right)^{2}\right. \\
& \left.+a\left(x_{2}-x_{1}\right)\left(y_{2}-y_{1}\right) H\left(x_{2}, x_{1}\right)\right\}, \\
& W_{4}=\left\{\gamma_{4} b(1-\beta) H\left(x_{2}, x_{1}\right)\left(x_{2}-x_{1}\right)^{2}+\xi_{3} \alpha a^{-1} F\left(z_{2}, z_{1}\right)\left(z_{2}-z_{1}\right)^{2}\right. \\
& \left.+b(1-\beta)\left(x_{2}-x_{1}\right)\left(z_{2}-z_{1}\right)\left[F\left(z_{2}, z_{1}\right)-a\right]\right\}, \\
& W_{5}=\left\{\gamma_{5} b(1-\beta) H\left(x_{2}, x_{1}\right)\left(x_{2}-x_{1}\right)^{2}+\eta_{3} a\left[G\left(y_{2}, y_{1}\right)-b(1-\beta)\right]\left(y_{2}-y_{1}\right)^{2}\right. \\
& \left.+b(1-\beta)\left(x_{2}-x_{1}\right)\left(y_{2}-y_{1}\right)\left[G\left(y_{2}, y_{1}\right)-b\right]\right\}, \\
& W_{6}=\left\{\xi_{4} \alpha a^{-1} F\left(z_{2}, z_{1}\right)\left(z_{2}-z_{1}\right)^{2}+\eta_{4} a\left[G\left(y_{2}, y_{1}\right)-b(1-\beta)\right]\left(y_{2}-y_{1}\right)^{2}\right. \\
& \left.+\left(1+\alpha a^{-1}\right)\left(y_{2}-y_{1}\right)\left(z_{2}-z_{1}\right)\left[G\left(y_{2}, y_{1}\right)-b\right]\right\}, \\
& W_{7}=\left\{\xi_{5} \alpha a^{-1} F\left(z_{2}, z_{1}\right)\left(z_{2}-z_{1}\right)^{2}+\eta_{5} a\left[G\left(y_{2}, y_{1}\right)-b(1-\beta)\right]\left(y_{2}-y_{1}\right)^{2}\right. \\
& \left.+a\left(y_{2}-y_{1}\right)\left(z_{2}-z_{1}\right)\left[F\left(z_{2}, z_{1}\right)-a\right]\right\}, \\
& W_{8}=\left\{b(1-\beta)\left(x_{2}-x_{1}\right)+a\left(y_{2}-y_{1}\right)+\left(1+\alpha a^{-1}\right)\left(z_{2}-z_{1}\right)\right\} \theta(t),
\end{aligned}
$$


with

$$
\begin{gathered}
F\left(z_{2}, z_{1}\right)=\frac{f\left(z_{2}\right)-f\left(z_{1}\right)}{z_{2}-z_{1}} \quad\left(z_{2} \neq z_{1}\right) \\
G\left(y_{2}, y_{1}\right)=\frac{g\left(y_{2}\right)-g\left(y_{1}\right)}{y_{2}-y_{1}} \quad\left(y_{2} \neq y_{1}\right) \\
H\left(x_{2}, x_{1}\right)=\frac{h\left(x_{2}\right)-h\left(x_{1}\right)}{x_{2}-x_{1}} \quad\left(x_{2} \neq x_{1}\right)
\end{gathered}
$$

and $\xi_{i}, \eta_{i}$, and $\gamma_{i},(i=1,2,3,4,5)$ are strictly positive constants such that

$$
\sum_{i=1}^{5} \xi_{i}=1, \quad \sum_{i=1}^{5} \eta_{i}=1, \quad \sum_{i=1}^{5} \gamma_{i}=1
$$

Also, let us denote $F\left(z_{2}, z_{1}\right), G\left(y_{2}, y_{1}\right)$, and $H\left(x_{2}, x_{1}\right)$ simply by $F, G$, and $H$, respectively. For strictly positive constants $k_{1}, k_{2}, k_{3}, k_{4}, k_{5}$, and $k_{6}$ conveniently chosen later, we get

$$
\begin{aligned}
(1+ & \left.\alpha a^{-1}\right)\left(x_{2}-x_{1}\right)\left(z_{2}-z_{1}\right) H \\
= & \left\{k_{1}\left(1+\alpha a^{-1}\right)^{1 / 2} H^{1 / 2}\left(x_{2}-x_{1}\right)+\frac{1}{2} k_{1}^{-1}\left(1+\alpha a^{-1}\right)^{1 / 2} H^{1 / 2}\left(z_{2}-z_{1}\right)\right\}^{2} \\
& -k_{1}^{2}\left(1+\alpha a^{-1}\right) H\left(x_{2}-x_{1}\right)^{2}-\frac{1}{4} k_{1}^{-2}\left(1+\alpha a^{-1}\right) H\left(z_{2}-z_{1}\right)^{2}, \\
a\left(x_{2}-x_{1}\right)\left(y_{2}-y_{1}\right) H & \\
= & \left\{k_{2} a^{1 / 2} H^{1 / 2}\left(x_{2}-x_{1}\right)+\frac{1}{2} k_{2}^{-1} a^{1 / 2} H^{1 / 2}\left(y_{2}-y_{1}\right)\right\}^{2} \\
& -k_{2}^{2} a H\left(x_{2}-x_{1}\right)^{2}-\frac{1}{4} k_{2}^{-2} a H\left(y_{2}-y_{1}\right)^{2}, \\
b(1- & \beta)\left(x_{2}-x_{1}\right)\left(z_{2}-z_{1}\right)[F-a] \\
= & \left\{\frac{1}{2} k_{3}^{-1} b^{1 / 2}(1-\beta)^{1 / 2}[F-a]^{1 / 2}\left(x_{2}-x_{1}\right)+k_{3} b^{1 / 2}(1-\beta)^{1 / 2}[F-a]^{1 / 2}\left(z_{2}-z_{1}\right)\right\}^{2} \\
& -\frac{1}{4} k_{3}^{-2} b(1-\beta)[F-a]\left(x_{2}-x_{1}\right)^{2}-k_{3}^{2} b(1-\beta)[F-a]\left(z_{2}-z_{1}\right)^{2}, \\
b(1- & \beta)\left(x_{2}-x_{1}\right)\left(y_{2}-y_{1}\right)[G-b] \\
= & \left\{k_{4} b^{1 / 2}(1-\beta)^{1 / 2}[G-b]^{1 / 2}\left(x_{2}-x_{1}\right)+\frac{1}{2} k_{4}^{-1} b^{1 / 2}(1-\beta)^{1 / 2}[G-b]^{1 / 2}\left(y_{2}-y_{1}\right)\right\}^{2} \\
& -k_{4}^{2} b(1-\beta)[G-b]\left(x_{2}-x_{1}\right)^{2}-\frac{1}{4} k_{4}^{-2} b(1-\beta)[G-b]\left(y_{2}-y_{1}\right)^{2},
\end{aligned}
$$


Discrete Dynamics in Nature and Society

$$
\begin{aligned}
(1+ & \left.\alpha a^{-1}\right)\left(y_{2}-y_{1}\right)\left(z_{2}-z_{1}\right)[G-b] \\
= & \left\{k_{5}\left(1+\alpha a^{-1}\right)^{1 / 2}[G-b]^{1 / 2}\left(y_{2}-y_{1}\right)+\frac{1}{2} k_{5}^{-1}\left(1+\alpha a^{-1}\right)^{1 / 2}[G-b]^{1 / 2}\left(z_{2}-z_{1}\right)\right\}^{2} \\
& -k_{5}^{2}\left(1+\alpha a^{-1}\right)[G-b]\left(y_{2}-y_{1}\right)^{2}-\frac{1}{4} k_{5}^{-2}\left(1+\alpha a^{-1}\right)[G-b]\left(z_{2}-z_{1}\right)^{2}, \\
a\left(y_{2}-y_{1}\right)\left(z_{2}-z_{1}\right)[F-a] & \\
= & \left\{\frac{1}{2} k_{6}^{-1} a^{1 / 2}[F-a]^{1 / 2}\left(y_{2}-y_{1}\right)+k_{6} a^{1 / 2}[F-a]^{1 / 2}\left(z_{2}-z_{1}\right)\right\}^{2} \\
& -\frac{1}{4} k_{6}^{-2} a[F-a]\left(y_{2}-y_{1}\right)^{2}-k_{6}^{2} a[F-a]\left(z_{2}-z_{1}\right)^{2}
\end{aligned}
$$

Thus,

$$
\begin{aligned}
W_{2}= & \left\{k_{1}\left(1+\alpha a^{-1}\right)^{1 / 2} H^{1 / 2}\left(x_{2}-x_{1}\right)+\frac{1}{2} k_{1}^{-1}\left(1+\alpha a^{-1}\right)^{1 / 2} H^{1 / 2}\left(z_{2}-z_{1}\right)\right\}^{2} \\
& +\left\{\gamma_{2} b(1-\beta) H-k_{1}^{2}\left(1+\alpha a^{-1}\right) H\right\}\left(x_{2}-x_{1}\right)^{2} \\
& +\left\{\xi_{2} \alpha a^{-1} F-\frac{1}{4} k_{1}^{-2}\left(1+\alpha a^{-1}\right) H\right\}\left(z_{2}-z_{1}\right)^{2}, \\
W_{3}= & \left\{k_{2} a^{1 / 2} H^{1 / 2}\left(x_{2}-x_{1}\right)+\frac{1}{2} k_{2}^{-1} a^{1 / 2} H^{1 / 2}\left(y_{2}-y_{1}\right)\right\}^{2} \\
& +\left\{\gamma_{3} b(1-\beta) H-k_{2}^{2} a H\right\}\left(x_{2}-x_{1}\right)^{2} \\
& +\left\{\eta_{2} a[G-b(1-\beta)]-\frac{1}{4} k_{2}^{-2} a H\right\}\left(y_{2}-y_{1}\right)^{2}, \\
W_{4}= & \left\{\frac{1}{2} k_{3}^{-1} b^{1 / 2}(1-\beta)^{1 / 2}[F-a]^{1 / 2}\left(x_{2}-x_{1}\right)+k_{3} b^{1 / 2}(1-\beta)^{1 / 2}[F-a]^{1 / 2}\left(z_{2}-z_{1}\right)\right\}^{2} \\
& +\left\{\gamma_{4} b(1-\beta) H-\frac{1}{4} k_{3}^{-2} b(1-\beta)[F-a]\right\}\left(x_{2}-x_{1}\right)^{2} \\
& +\left\{\xi_{3} \alpha a^{-1} F-k_{3}^{2} b(1-\beta)[F-a]\right\}\left(z_{2}-z_{1}\right)^{2}, \\
& +\left\{\eta_{3} a[G-b(1-\beta)]-\frac{1}{4} k_{4}^{-2} b(1-\beta)[G-b]\right\}\left(y_{2}-y_{1}\right)^{2}, \\
& +\left\{\gamma_{5} b(1-\beta) H-k_{4}^{2} b(1-\beta)[G-b]\right\}\left(x_{2}-x_{1}\right)^{2} \\
W_{5}= & \left\{k_{4} b^{1 / 2}(1-\beta)^{1 / 2}[G-b]^{1 / 2}\left(x_{2}-x_{1}\right)+\frac{1}{2} k_{4}^{-1} b^{1 / 2}(1-\beta)^{1 / 2}[G-b]^{1 / 2}\left(y_{2}-y_{1}\right)\right\}^{2} \\
& \\
&
\end{aligned}
$$




$$
\begin{aligned}
W_{6}= & \left\{k_{5}\left(1+\alpha a^{-1}\right)^{1 / 2}[G-b]^{1 / 2}\left(y_{2}-y_{1}\right)+\frac{1}{2} k_{5}^{-1}\left(1+\alpha a^{-1}\right)^{1 / 2}[G-b]^{1 / 2}\left(z_{2}-z_{1}\right)\right\}^{2} \\
& +\left\{\eta_{4} a[G-b(1-\beta)]-k_{5}^{2}\left(1+\alpha a^{-1}\right)[G-b]\right\}\left(y_{2}-y_{1}\right)^{2} \\
& +\left\{\xi_{4} \alpha a^{-1} F-\frac{1}{4} k_{5}^{-2}\left(1+\alpha a^{-1}\right)[G-b]\right\}\left(z_{2}-z_{1}\right)^{2}, \\
W_{7}= & \left\{\frac{1}{2} k_{6}^{-1} a^{1 / 2}[F-a]^{1 / 2}\left(y_{2}-y_{1}\right)+k_{6} a^{1 / 2}[F-a]^{1 / 2}\left(z_{2}-z_{1}\right)\right\}^{2} \\
& +\left\{\eta_{5} a[G-b(1-\beta)]-\frac{1}{4} k_{6}^{-2} a[F-a]\right\}\left(y_{2}-y_{1}\right)^{2} \\
& +\left\{\xi_{5} \alpha a^{-1} F-k_{6}^{2} a[F-a]\right\}\left(z_{2}-z_{1}\right)^{2} .
\end{aligned}
$$

Moreover, in view of (3.2), we obtain for all $x_{i}, z_{i}(i=1,2)$ in $\Re$,

$$
W_{2} \geq 0
$$

if

$$
k_{1}^{2} \leq \frac{\gamma_{2}(1-\beta) a b}{(\alpha+a)} \text { with } H \leq \frac{4 \xi_{2} \gamma_{2} \alpha(1-\beta) a^{2} b}{(\alpha+a)^{2}}
$$

and for all $x_{i}, y_{i}(i=1,2)$ in $\Re$,

$$
W_{3} \geq 0
$$

if

$$
k_{2}^{2} \leq \frac{\gamma_{3}(1-\beta) b}{a} \quad \text { with } H \leq \frac{4 \eta_{2} \gamma_{3} \beta(1-\beta) b^{2}}{a} .
$$

Combining all the inequalities in (3.16) and (3.18), we have for all $x_{i}, y_{i}, z_{i}(i=1,2)$ in $\mathfrak{R}$,

$$
W_{2} \geq 0, \quad W_{3} \geq 0,
$$

if

$$
H \leq k a b \quad \text { with } k=\min \left\{\frac{4 \xi_{2} \gamma_{2} \alpha(1-\beta) a}{(\alpha+a)^{2}}, \frac{4 \eta_{2} \gamma_{3} \beta(1-\beta) b}{a^{2}}\right\}<1 .
$$

Also, for all $x_{i}, z_{i}(i=1,2)$ in $\Re$,

$$
W_{4} \geq 0 \text {, }
$$


if

$$
\frac{a_{0}-a}{4 \gamma_{4} \delta} \leq k_{3}^{2} \leq \frac{\xi_{3} \alpha}{(1-\beta) b\left(a_{0}-a\right)}
$$

for all $x_{i}, y_{i}(i=1,2)$ in $\Re$,

$$
W_{5} \geq 0,
$$

if

$$
\frac{(1-\beta)\left(b_{0}-b\right)}{4 \beta a \eta_{3}} \leq k_{4}^{2} \leq \frac{\delta \gamma_{5}}{\left(b_{0}-b\right)}
$$

for all $y_{i}, z_{i}(i=1,2)$ in $\Re$,

$$
W_{6} \geq 0,
$$

if

$$
\frac{(\alpha+a)\left(b_{0}-b\right)}{4 \xi_{4} \alpha a} \leq k_{5}^{2} \leq \frac{\eta_{4} \beta b a^{2}}{(\alpha+a)\left(b_{0}-b\right)},
$$

and for all $y_{i}, z_{i}(i=1,2)$ in $\Re$,

$$
W_{7} \geq 0
$$

if

$$
\frac{a_{0}-a}{4 \eta_{5} \beta b} \leq k_{6}^{2} \leq \frac{\xi_{5} \alpha}{a\left(a_{0}-a\right)} .
$$

Further

$$
W_{1} \geq 2 D_{10}\left\{\left(x_{2}-x_{1}\right)^{2}+\left(y_{2}-y_{1}\right)^{2}+\left(z_{2}-z_{1}\right)^{2}\right\}
$$

where $2 D_{10}=\min \left\{\gamma_{1} b \delta(1-\beta), \eta_{1} a b \beta, \xi_{1} \alpha\right\}$, on the other hand

$$
W_{8} \leq D_{11}\left\{\left(x_{2}-x_{1}\right)^{2}+\left(y_{2}-y_{1}\right)^{2}+\left(z_{2}-z_{1}\right)^{2}\right\}^{1 / 2}|\theta(t)|,
$$

where $D_{11}=2 \max \left\{b(1-\beta), a,\left(1+\alpha a^{-1}\right)\right\}$. 
Bringing together the estimates just obtained for $W_{1}, W_{2}, W_{3}, W_{4}, W_{5}, W_{6}, W_{7}$, and $W_{8}$ in (3.10) and using (3.8), we have

$$
\frac{d W}{d t} \leq-2 D_{10} S(t)+D_{11} S^{1 / 2}(t)|\theta(t)|
$$

This completes the proof of Lemma 3.2.

\section{Proof of Theorem 2.2}

This follows directly from [5], on using inequality (3.32). Let $v$ be any constant in the range $1 \leq v \leq 2$. Set $2 \mu=2-v$, so that $0 \leq \mu \leq 1 / 2$. We rewrite (3.32) in the form

$$
\frac{d W}{d t}+D_{10} S \leq-D_{10} S+D_{11} S^{1 / 2}|\theta|=D_{11} S^{\mu} W^{*},
$$

where

$$
W^{*}=\left(|\theta|-D_{12} S^{1 / 2}\right) S^{1 / 2-\mu}
$$

with $D_{12}=D_{10} D_{11}^{-1}$, considering the two cases

(i) $|\theta|<D_{12} S^{1 / 2}$ and

(ii) $|\theta| \geq D_{12} S^{1 / 2}$

separately. If $|\theta|<D_{12} S^{1 / 2}$, then $W^{*}<0$. On the other hand, if $|\theta| \geq D_{12} S^{1 / 2}$, then the definition of $W^{*}$ in (4.2) gives at least

$$
W^{*} \leq S^{(1 / 2-\mu)}|\theta|
$$

and also $S^{1 / 2} \leq|\theta| / D_{12}$. This implies that

$$
S^{1 / 2(1-2 \mu)} \leq\left[\frac{|\theta|}{D_{12}}\right]^{(1-2 \mu)}
$$

Therefore

$$
S^{1 / 2(1-2 \mu)}|\theta| \leq\left[\frac{|\theta|}{D_{12}}\right]^{(1-2 \mu)} \times|\theta|,
$$

from which together with $W^{*}$, we obtain

$$
W^{*} \leq D_{13}|\theta|^{2(1-\mu)},
$$


where $D_{13}=D_{12}^{(2 \mu-1)}$. Again due to (4.1) and using the estimate on $W^{*}$ for $W^{*}$, we have

$$
\begin{aligned}
\frac{d W}{d t}+D_{10} S & \leq D_{11} D_{13} S^{\mu}|\theta|^{2(1-\mu)} \\
& \leq D_{14} S^{\mu} \phi^{2(1-\mu)} S^{(1-\mu)}
\end{aligned}
$$

where $D_{14}=3^{1-\mu} D_{11} D_{13}$, which follows from

$$
\begin{aligned}
|\theta| & =\left|p\left(t, x_{2}, y_{2}, z_{2}\right)-p\left(t, x_{1}, y_{1}, z_{1}\right)\right| \\
& \leq \phi(t)\left\{\left|x_{2}-x_{1}\right|+\left|y_{2}-y_{1}\right|+\left|z_{2}-z_{1}\right|\right\} .
\end{aligned}
$$

In view of the fact that $v=2(1-\mu)$, we obtain

$$
\frac{d W}{d t} \leq-D_{10} S+D_{14} \phi^{v} S
$$

and on using inequality (3.4), we have

$$
\frac{d W}{d t}+\left(D_{15}-D_{16} \phi^{v}(t)\right) W \leq 0
$$

for some positive constants $D_{15}$ and $D_{16}$. On integrating (4.10) from $t_{1}$ to $t_{2} \quad\left(t_{2} \geq t_{1}\right)$, we have

$$
W\left(t_{2}\right) \leq W\left(t_{1}\right) \exp \left\{-D_{15}\left(t_{2}-t_{1}\right)+D_{16} \int_{t_{2}}^{t_{2}} \phi^{v}(\tau) d \tau\right\} .
$$

Again, using Lemma 3.1, we obtain (2.4), with $D_{2}=D_{7} D_{6}^{-1}, D_{3}=D_{15}$, and $D_{4}=D_{16}$. This completes the proof of Theorem 2.2.

\section{Proof of Theorem 2.1}

This follows from the estimate (2.4) and the condition (2.3) on $\phi(t)$. Choose $D_{1}=D_{3} D_{4}^{-1}$ in (2.3). From the estimate (2.4), if

$$
\int_{t_{1}}^{t_{2}} \phi^{v}(\tau) d \tau \leq D_{3} D_{4}^{-1}\left(t_{2}-t_{1}\right)
$$

then the exponential index remains negative for all $t_{2}-t_{1} \geq 0$. Then, as $t=\left(t_{2}-t_{1}\right) \rightarrow \infty$, we have $S(t) \rightarrow 0$, and this gives

$$
x_{2}-x_{1} \longrightarrow 0, \quad y_{2}-y_{1} \longrightarrow 0, \quad z_{2}-z_{1} \longrightarrow 0,
$$

as $t \rightarrow \infty$. This completes the proof of Theorem 2.1. 


\section{Acknowledgment}

The author would like to express sincere thanks to the anonymous referees for their invaluable corrections, comments, and suggestions.

\section{References}

[1] A. M. Liapunov, Stability of Motion, Academic Press, New York, NY, USA, 1966.

[2] A. U. Afuwape and M. O. Omeike, "Convergence of solutions of certain non-homogeneous third order ordinary differential equations," Kragujevac Journal of Mathematics, vol. 31, pp. 5-16, 2008.

[3] A. U. Afuwape and M. O. Omeike, "Further ultimate boundedness of solutions of some system of third order nonlinear ordinary differential equations," Acta Universitatis Palackianae Olomucensis. Facultas Rerum Naturalium. Mathematica, vol. 43, pp. 7-20, 2004.

[4] J. O. C. Ezeilo, "A note on the convergence of solutions of certain second order differential equations," Portugaliae Mathematica, vol. 24, pp. 49-58, 1965.

[5] J. O. C. Ezeilo, "New properties of the equation $\dddot{x}+a \ddot{x}+b \dot{x}+h(x)=P(t, x, \dot{x}, \ddot{x})$ for certain special values of the incrementary ratio $y^{-1}\{h(x+y)-h(x)\}, "$ in Équations différentielles et fonctionnelles non linéaires (Actes Conférence Internat. "Equa-Diff 73", Brussels/Louvain-la-Neuve, 1973), P. Janssens, J. Mawhin, and N. Rouche, Eds., pp. 447-462, Hermann, Paris, France, 1973.

[6] F. W. Meng, "Ultimate boundedness results for a certain system of third order nonlinear differential equations," Journal of Mathematical Analysis and Applications, vol. 177, no. 2, pp. 496-509, 1993.

[7] H. O. Tejumola, "On the convergence of solutions of certain third-order differential equations," Annali di Matematica Pura ed Applicata, vol. 78, no. 1, pp. 377-386, 1968.

[8] H. O. Tejumola, "Convergence of solutions of certain ordinary third order differential equations," Annali di Matematica Pura ed Applicata, vol. 94, no. 1, pp. 247-256, 1972.

[9] C. Tunç, "Boundedness of solutions of a third-order nonlinear differential equation," Journal of Inequalities in Pure and Applied Mathematics, vol. 6, no. 1, article 3, pp. 1-6, 2005.

[10] C. Tunç and E. Tunç, "New ultimate boundedness and periodicity results for certain third-order nonlinear vector differential equations," Mathematical Journal of Okayama University, vol. 48, pp. 159_ $172,2006$.

[11] C. Tunç and E. Tunç, "On the asymptotic behavior of solutions of certain second-order differential equations," Journal of the Franklin Institute, vol. 344, no. 5, pp. 391-398, 2007.

[12] M. O. Omeike, "New result in the ultimate boundedness of solutions of a third-order nonlinear ordinary differential equation," Journal of Inequalities in Pure and Applied Mathematics, vol. 9, no. 1, article 15, pp. 1-8, 2008.

[13] R. Reissig, G. Sansone, and R. Conti, Non-Linear Differential Equations of Higher Order, Noordhoff International, Leyden, The Netherlands, 1974.

[14] T. Yoshizawa, Stability Theory by Liapunov's Second Method, Publications of the Mathematical Society of Japan, no. 9, The Mathematical Society of Japan, Tokyo, Japan, 1966. 


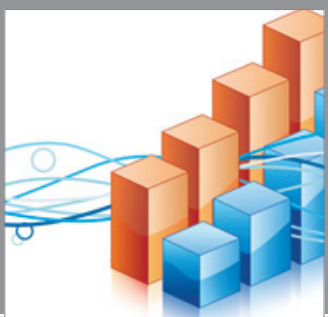

Advances in

Operations Research

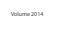

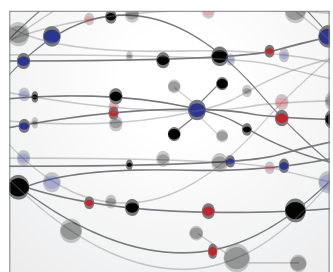

\section{The Scientific} World Journal
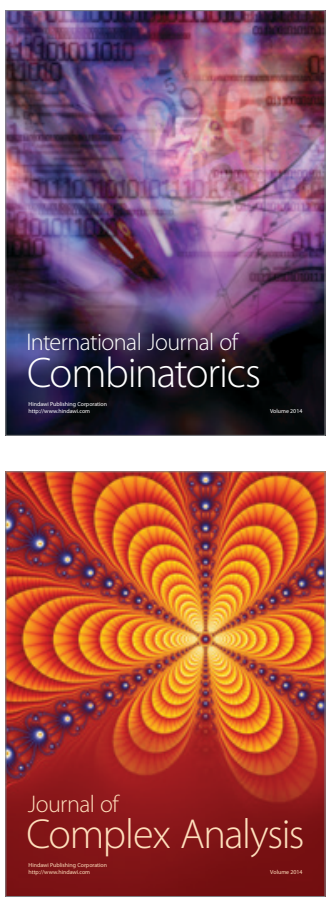

International Journal of

Mathematics and

Mathematical

Sciences
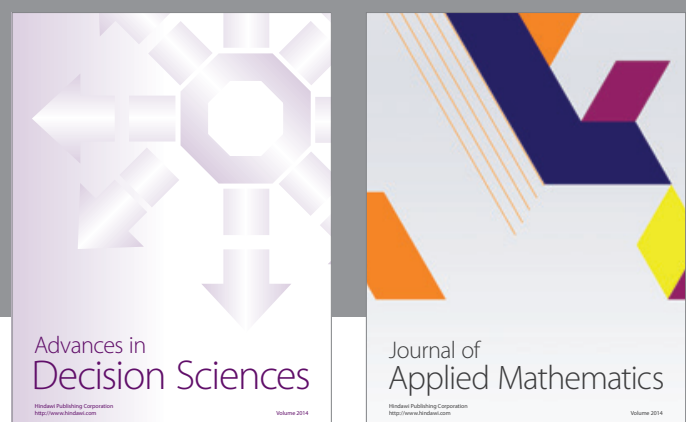

Journal of

Applied Mathematics
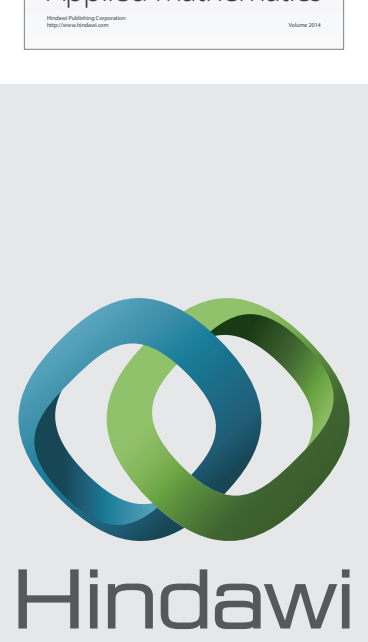

Submit your manuscripts at http://www.hindawi.com
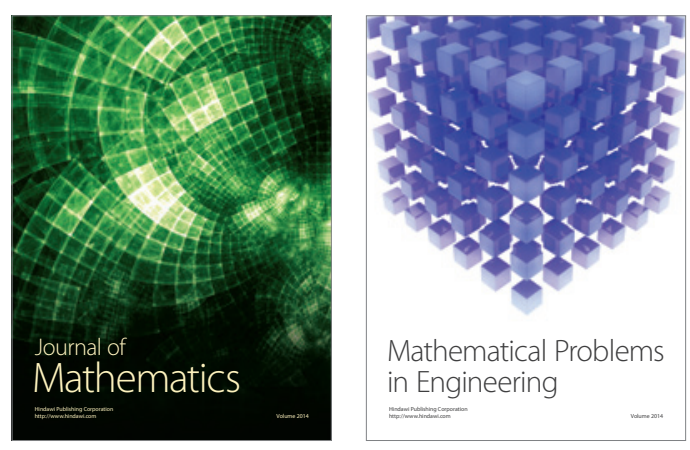

Mathematical Problems in Engineering
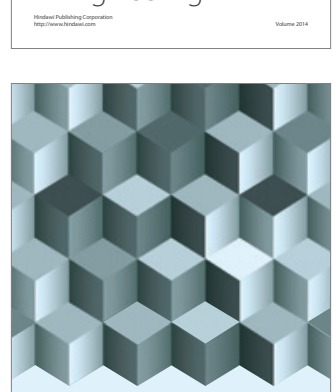

Journal of

Function Spaces
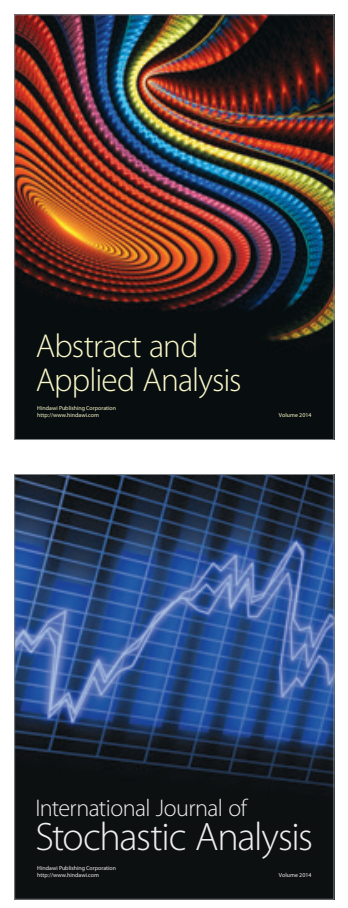

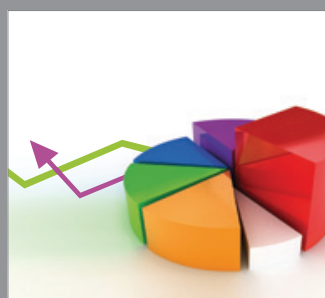

ournal of

Probability and Statistics

Promensencen
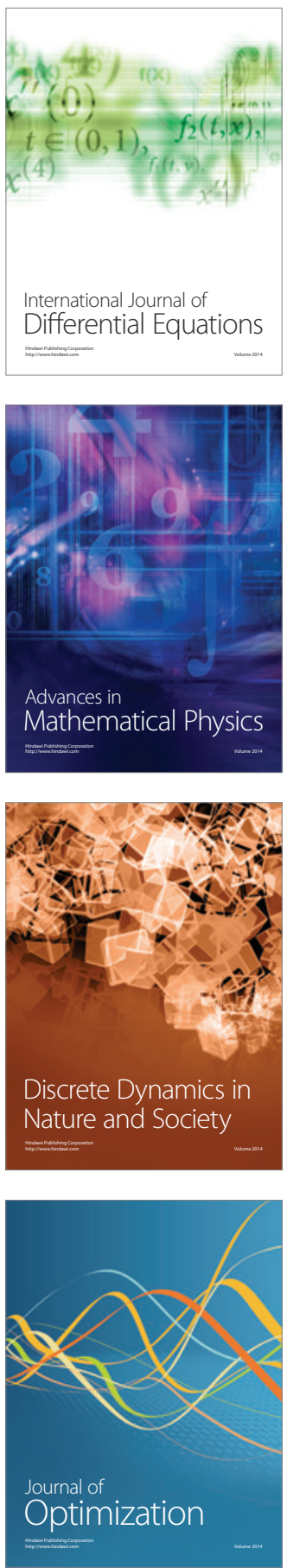